\title{
Tecnologia educacional para a prevenção de doenças em crianças pré-escolares e escolares
}

\author{
Educational technology for disease prevention in preschoolers and schoolchildren
}

Tecnología educativa para la prevención de enfermedades en preescolares y escolares

\section{RESUMO}

Objetivo: Relatar a experiência acerca da criação e aplicação de uma tecnologia educacional de enfermagem voltada para a lavagem das mãos como forma de prevenção de doenças entre pré-escolares e escolares. Método: Estudo descritivo do tipo relato de experiência, realizado por acadêmicos do curso de Enfermagem, durante o estágio supervisionado. Resultados: A experiência oportunizou o aprendizado sobre a elaboração de uma tecnologia educacional voltada para o público infantil utilizando ferramentas atrativas para a idade. A aplicação de uma atividade educativa planejada foi essencial ao processo de formação como enfermeiros, sendo essa experiência positiva, para auxiliar no planejamento de futuras intervenções educacionais, utilizando ferramentas educativas de acordo com o público-alvo. Conclusão: Essa experiência foi positiva, para auxiliar no planejamento de futuras intervenções educacionais, utilizando ferramentas educativas de acordo com o público-alvo.

Descritores: Saúde da criança; Educação em saúde; Tecnologia educacional; Serviços de saúde escolar; Enfermagem.

\section{ABSTRACT}

Purpose: To report the experience creating and applying an educational technology of nursing focused on hand washing to prevent diseases among preschoolers and schoolchildren. Method: A descriptive study of the experience report given by nursing undergraduate students during the supervised training. Results: The experience provided the opportunity to learn about the development of educational technologies for children, using attractive tools for their age. The implementation of a planned educational activity was essential in the nurses' training process. Furthermore, this experience provided means to aid the planning of future educational interventions using educational tools according to the target audience. Conclusion: This experience was positive to assist in the planning of future educational interventions using educational tools according to the target audience.

Descriptors: Child health; Health education; Educational technology; School health services; Nursing.

\section{RESUMEN}

Objetivo: Relatar la experiencia sobre la creación y aplicación de una tecnología educativa de enfermería orientada al lavado de manos como forma de prevención de enfermedades entre preescolares y escolares. Método: Estudio descriptivo del tipo relato de experiencia realizado por académicos del curso de Enfermería durante la etapa supervisada. Resultados: La experiencia dio oportunidad al aprendizaje sobre la elaboración de una tecnología educativa orientada al público infantil utilizando herramientas atractivas para la edad. La aplicación de una actividad educativa planificada fue esencial en el proceso de formación de enfermeros, siendo esa experiencia positiva para auxiliar en la organización de futuras intervenciones educativas utilizando herramientas didácticas de acuerdo con el público al que van destinadas. Conclusión: Esta experiencia fue positiva para ayudar en la planificación de futuras intervenciones educativas utilizando herramientas didácticas de acuerdo con el público objetivo.

Descriptores: Salud del Niño; Educación en Salud; Tecnología Educacional; Servicios de Salud Escolar; Enfermería.

Emanuel Adenilton Teixeira

Barbosa $^{1}$

0000-0002-3628-4039

Vanderlene Mota Andrade ${ }^{1}$

0000-0003-3032-2417

Talita Almeida de Oliveira ${ }^{1}$

0000-0001-5284-5927

Maria Corina Amaral Viana ${ }^{2}$

(D) 0000-0002-6890-9400

Edna Maria Camelo Chaves ${ }^{3}$

0000-0001-9658-0377

Aliniana da Silva Santos
0000-0002-1742-2758

${ }^{1}$ Centro Universitário UniFanor, Fortaleza, CE, Brasil.

2 Universidade Regional do Cariri, Fortaleza, CE, Brasil.

3 Universidade Estadual do Ceará Fortaleza, CE, Brasil.

Autor correspondente:

Aliniana da Silva Santos

E-mail: enfa.aliniana@gmail.com

\section{Como citar este artigo:}

Barbosa EAT, Andrade VM, Oliveira, $\mathrm{TA}$, et al. Tecnologia educacional para prevenção de doenças em crianças pré-escolares e escolares. Revista de Enfermagem do Centro-Oeste Mineiro. 2021;11:e3094. [Access Available in: DOI: http://dx.doi.org/10.19175/recom.v11i $\underline{0.3094}$ 


\section{INTRODUÇÃO}

As doenças mais comuns na infância são as infecções respiratórias e enteroparasitárias, as quais são as mais prevalentes entre pré-escolares e escolares ${ }^{(1)}$. Um terço da população mundial, principalmente nos países em desenvolvimento, está infectada com helmintos transmitidos pelo solo, que são vermes parasitas nematoides intestinais, entre eles, as lombrigas (Ascaris lumbricoides) se destacam, sendo os maiores e mais prevalentes helmintos transmitidos, responsável por 1 bilhão de infecções e quase metade da carga global de doenças é suportada por crianças dos 5 aos 14 anos de idade ${ }^{(2)}$.

As crianças entre 4 e 7 anos ainda não possuem noções de higiene formadas, aumentando consideravelmente os riscos de infecção, nessa faixa etária, somado ao sistema imunológico deficiente ${ }^{(1-4)}$. Verifica-se que a principal forma de prevenção contra essas infecções ocorre, sobretudo, pela lavagem das mãos, assim, medidas de prevenção simples precisam ser adotadas. A literatura destaca a importância de intervenções educativas, voltadas aos pré-escolares e escolares, para o incentivo à lavagem das mãos, a fim de reduzir as infecções gastrointestinais ${ }^{(5)}$.

No cenário internacional, um estudo de intervenção educativa, na India, realizado a partir de um ensaio randomizado sobre a lavagem das mãos direcionadas aos escolares de 5 anos de idade e seus familiares, promoveu a conscientização acerca dos germes, como eles se espalhavam e o seu papel na doença, obtendo como desfecho a redução dos episódios de diarreia, Infecções Respiratórias, entre outras, bem como queda do absenteísmo escolar, trazendo assim evidências importantes sobre a lavagem das mãos para a prevenção de doenças prevalentes na infância ${ }^{(6)}$.

A infância é considerada um período crucial de aquisição de novos conhecimentos e hábitos, sendo as escolas de educação infantil espaços privilegiados para intervenções educativas direcionadas à promoção da saúde com vista à adoção de comportamentos saudáveis desde a infância ${ }^{(7)}$. Cuidados básicos com o corpo, como tomar banho, escovar os dentes e lavar as mãos ajuda na prevenção de doenças, devendo os hábitos de higiene na escola serem fortalecidos ${ }^{(8)}$.

Destaca-se a educação em saúde como ferramenta necessária à promoção da saúde na infância. Entre os processos educativos, destacam-se as tecnologias educacionais (TE) que são definidas como a sistematização do conhecimento a partir do planejamento e acompanhamento do processo educacional ${ }^{(9)}$. A utilização dessas tecnologias se dá baseada na elaboração planejada de uma ou várias estratégias educativas, que, no caso do público infantil, deve ter um caráter lúdico. As intervenções educacionais devem ocorrer de maneira descontraída e participativa, destacando-se assim práticas lúdicas com crianças pré-escolares como efetivas para a melhoria dos comportamentos direcionados à saúde ${ }^{(7)}$. Acredita-se que, por meio da TE, é possível dinamizar o processo de ensinoaprendizagem, com a utilização de materiais voltados ao desenvolvimento socioeducativo das crianças.

Comumente, as crianças não realizam a higiene das mãos, muitas vezes, por falta de orientação familiar, ou na própria escola, por isso, a importância de programar ações educativas direcionadas ao público infantil, tendo como cenário de intervenção a escola.

Dessa forma, o trabalho tem como objetivo relatar a experiência acerca da criação e aplicação de uma Tecnologia Educacional de Enfermagem, voltada para a lavagem das mãos, como forma de prevenção de doenças entre pré-escolares e escolares.

\section{MÉTODO}

Trata-se de um estudo descritivo do tipo relato de experiência, realizado por acadêmicos do curso de Enfermagem, durante o estágio supervisionado I, no período de maio de 2018, em uma escola infantil, localizada na Regional II, no Bairro Papicu, na cidade de Fortaleza, Ceará.

A ação foi realizada em uma instituição de educação infantil por ser considerada um espaço privilegiado para a promoção da saúde da criança $^{(8)}$ na faixa etária 4 a 7 anos de idade. A educação em saúde foi autorizada pela coordenação da instituição. Participaram da intervenção educativa crianças do ensino integral do infantil I ao III. A ação aconteceu na quadra da escola por ser um local estratégico próximo a vários lavadouros.

Alguns pesquisadores deste trabalho apresentam expertise na área de saúde da criança tendo como foco ações educativas utilizando TE, com várias publicações em periódicos nacionais e internacionais na área. Os acadêmicos de enfermagem foram incentivados a elaborar uma 
ação educativa, por meio de planejamento de forma criativa e lúdica, direcionada às reais necessidades do público-alvo. Para escolher o assunto que seria trabalhado com as crianças, os discentes de enfermagem realizaram um levantamento junto à direção da escola escolhida, por conveniência, acerca dos assuntos que poderiam ser trabalhados com as crianças, entre eles, surgiu a lavagem das mãos.

A Tecnologia Educacional de Enfermagem foi composta por recursos visuais divididos em 3 etapas:

1) Em uma roda, foi realizada a exposição do problema de forma lúdica sobre a temática (momentos em que se deve lavar as mãos), utilizando roda de conversa e, como recurso didático, foram utilizadas pequenas placas contendo desenhos de germes. Essas figuras foram encontradas no google imagem, impressas em folha A4 colorida e pregadas em hastes de madeira, conforme Figura 1 apresentada nos resultados.

Ainda nessa etapa, foi simulado o passo a passo da higienização das mãos preconizado pela ANVISA(10) p.30-36 ao citar: "1의 Abrir a torneira e molhar as mãos; 2ㅇ) Aplicar na palma da mão quantidade suficiente de sabonete líquido; 3ㅇ) Ensaboar as palmas das mãos, friccionando-as entre si; 4의) Esfregar a palma da mão direita contra o dorso da mão esquerda, entrelaçando os dedos e vice-versa; 50) Entrelaçar os dedos e friccionar os espaços interdigitais; 6ㅇ) Esfregar o polegar direito com o auxílio da palma da mão esquerda, realizando movimento circular e viceversa; 70) Friccionar as polpas digitais (ponta dos dedos) e as unhas da mão esquerda contra a palma da mão direita, fechada em concha, fazendo movimento circular e vice-versa; 8ㅇ) Esfregar o punho esquerdo com o auxílio da palma da mão direita, realizando movimento circular e vice-versa; 9ㅇ) Enxaguar as mãos, retirando os resíduos de sabonete; 10) Secar as mãos com papel toalha descartável, iniciando pelas mãos e seguindo pelos punhos".

2) Dinâmica explicando como os microorganismos são passados pelas mãos, utilizando como ferramentas de ensino: luvas, balões e tinta guache e devidamente descritas no resultado desta pesquisa.

3) Oficina educativa para a higienização adequada das mãos, utilizando sabonete líquido e lavadouros. Nessa etapa, cada criança foi convidada a lavar as mãos, utilizando os conhecimentos que adquiriu durante a ação educativa.

A estratégia dos balões e tintas foi vista pelos discentes, em programa educativo na faculdade, sendo adaptada a proposta da pesquisa, utilizando as figuras ilustrativas. Assim, a tecnologia educacional deste estudo foi produzida com recursos visuais, como ilustrações e materiais já existentes, adaptados a uma nova abordagem, direcionada ao incentivo da higienização das mãos pelas crianças.

\section{RESULTADOS E DISCUSSÃO}

A experiência oportunizou o aprendizado acerca da elaboração de uma tecnologia educacional, voltada para o público infantil, utilizando linguagem simples e recursos visuais atrativos para a idade. As etapas realizadas estão descritas a seguir:

A primeira etapa teve como estratégia educativa uma roda de conversa do tipo expositivo-participativa com as crianças sentadas no chão, em forma de um círculo, em que os discentes demonstraram a técnica correta da higienização das mãos, seguindo o passo a passo conforme Anvisa ${ }^{(10)}$. Utilizou-se álcool em gel para a demonstração dos passos. Foram utilizados desenhos ilustrativos, representando os microorganismos (vermes), conforme mostra a Figura 1. As figuras foram passadas entre as crianças no círculo no intuito de explicar-Ihes como ocorria a transmissão das doenças e quais as medidas de prevenção na intenção de interagir com elas, utilizando essas figuras em forma de desenhos animados, como uma maneira de facilitar o seu processo de entendimento acerca dos microorganismos que são invisíveis aos olhos, mas que estão presentes nas mãos quando não higienizadas.

Figura 1 - Representação dos micro-organismos utilizados na tecnologia educacional de enfermagem, Fortaleza, CE, Brasil, 2018.

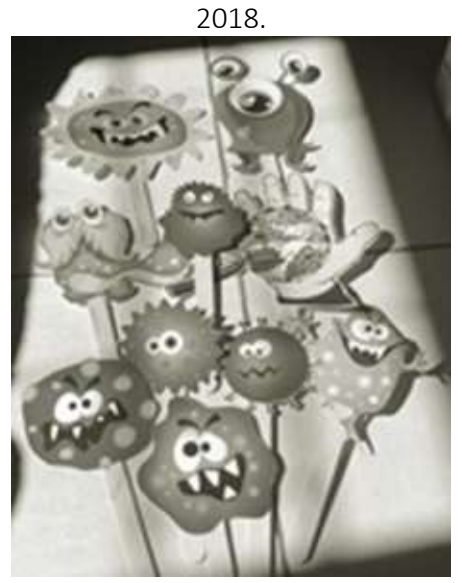

Fonte: Google Imagens adaptado (2018). 
$\mathrm{Na}$ segunda etapa, foi utilizada como estratégia educativa uma dinâmica com as crianças ainda sentadas e dispostas em círculo, as quais foram calçadas luvas de procedimento tamanho $P$ nas suas mãos, utilizando as ferramentas ilustradas na Figura 2.

Figura 2 - Ferramentas educativas utilizadas na dinâmica sobre a contaminação das mãos pelos micro-organismos, Fortaleza, Ceará, Brasil, 2018.

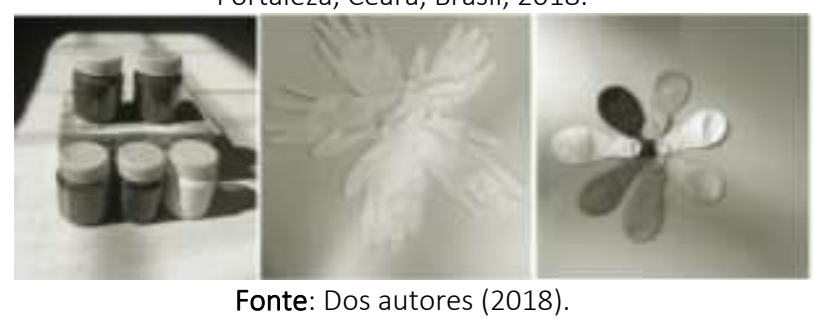

Após calçar as luvas nas mãos das crianças, foi utilizada tinta guache de cores diferentes (cada cor simbolizava um micro-organismo) que foi passada nas luvas. Após essa aplicação, um balão cheio de ar foi repassado entre as crianças com suas mãos enluvadas e com tinta. Ao final da dinâmica, o balão estava repleto de marcas de tinta de diferentes cores. Assim, foi possível demonstrar para as crianças como os microrganismos são passados de uma pessoa para outra ou do alimento para o nosso organismo por intermédio das mãos, podendo, assim, demonstrar de forma lúdica que, quando não se lavam as mãos, os microrganismos passam de uma pessoa para a outra, causando várias doenças, como dor de barriga (diarreia), resfriado, gripe, entre outras.

Por fim, foi realizada a terceira etapa, a oficina de higienização das mãos, na qual as crianças foram levadas aos lavadouros para a implementação da técnica de lavagem e secagem das mãos de forma correta, conforme foi mostrado na primeira etapa da atividade educativa. Foi possível perceber que a maioria conseguiu aprender os passos demonstrados.

A tecnologia educacional de enfermagem mostrou-se positiva e apropriada para a faixa etária, uma vez que foram utilizadas ferramentas educacionais de uso frequente pelas crianças (tinta guache e balões), que pudessem mostrar a importância de higienizar as mãos.

A escola é um ambiente ideal para realizar o encontro da educação e da saúde, por ser um espaço propício à construção de relações favoráveis à promoção da saúde. A tarefa, ainda incompleta, é de integrar saberes do currículo educacional de forma transversal, como estratégia pedagógica para que a educação em saúde no ambiente escolar seja crítica e promova a consciência sanitária ${ }^{(11)}$. Dessa forma, o Programa Saúde na Escola (PSE) constitui uma estratégia, para complementar a necessidade do fortalecimento da integração entre os setores educação e saúde, promovendo a intersetorialidade promulgada pelo SUS e a corresponsabilização entre estes setores, habituados a trabalhar isoladamente ${ }^{(12)}$.

Em um estudo de revisão sistemática, foi possível identificar que intervenção educativa, voltada para a higienização das mãos, é capaz de reduzir a incidência de diarreia e doenças respiratórias, reduzindo assim 0 absenteísmo escolar principalmente nos países em países em desenvolvimento ${ }^{(13)}$. As ações educativas acerca da vantagem de lavar as mãos tem como propósito amenizar o número de infecções intestinais causadas por helmintos que comumente infectam o público infantil ${ }^{(14)}$.

As crianças, em especial os pré-escolares e escolares, possuem capacidade neurológica de captar informações, sendo fundamental, nesse período de desenvolvimento, estimular o seu aprendizado, utilizando a linguagem do mundo infantil, por meio da utilização de estratégias lúdicas, para favorecer-lhes um link entre 0 conhecimento e sua aplicação na prática de vida diária. A implementação de um pacote educacional pode resultar na prevenção da infecção por helmintos transmitidos pelo solo, uma vez que o aumento no conhecimento pode levar a melhores práticas de higiene, adotando um novo comportamento que resulte assim em menos infecções por esses parasitas ${ }^{(1)}$.

Durante a infância, o mundo da aprendizagem é permeado pela descontração, brincadeira, pelo lúdico, oportunizando que a criança aprenda brincando e torne esse processo de ensino-aprendizagem prazeroso. Atividades lúdicas, como forma de aprendizagem, são importantes para promover o conhecimento de forma dinâmica, sendo considerada favorável para um ambiente motivador e agradável, possibilitando a aprendizagem, além de reforçar ideias pré-estabelecidas ${ }^{(15-16)}$. Por meio da brincadeira, a criança desenvolve seus sentidos e alcança inúmeras habilidades ${ }^{(16)}$.

A educação em saúde com um enfoque preventivo pode promover o controle de muitas doenças transmitidas pela falta de higienização das mãos. Uma das maneiras de se conseguir 
vencer a expansão dos micro-organismos é a mobilização da comunidade escolar, porque esse público está em processo de formação do conhecimento(17).

Nesse contexto, torna-se necessária a participação de educadores que estejam dispostos a implementar ações, para o desenvolvimento saudável da criança, atuando principalmente no âmbito da promoção da saúde. O enfermeiro é um importante ator no processo educativo, podendo atuar de forma complementar para o desenvolvimento integral da criança nos ambientes escolares ${ }^{(18)}$.

A literatura ressalta a necessidade de integração entre profissionais de saúde, educação e sociedade, a fim de favorecer a construção de condutas voltadas para a promoção da saúde das crianças na fase escolar, indicando a escola como espaço em destaque para que ocorram as mudanças de comportamentos de risco ${ }^{(19)}$.

O enfermeiro, entre os profissionais de saúde, desempenha um papel fundamental, no desenvolvimento do processo de ensinoaprendizagem, por estar diretamente envolvido com o manejo e sistematização do cuidado, em diferentes cenários, principalmente, no que diz respeito à atenção primária à saúde ${ }^{(20)}$. O cuidar e o educar são atribuições indissociáveis, no processo de trabalho da enfermagem, sendo o enfermeiro essencial junto à equipe na construção e na reestruturação do modelo de atenção à saúde ${ }^{(21)}$.

Assim, a elaboração de tecnologias educacionais por parte dos acadêmicos em enfermagem serve tanto para a sua capacitação, para futuras elaborações de intervenções educativas, como serviu para reforçar a capacidade individual e coletiva das crianças que são consideradas importantes e necessárias para que haja a redução de doenças causadas pela falta da higienização das mãos.

\section{CONSIDERAÇÕES FINAIS}

A aplicação de uma atividade educativa planejada foi essencial no processo de formação acadêmica, sendo que é uma experiência positiva para auxiliar no planejamento de futuras intervenções educacionais utilizando ferramentas lúdicas entre o público infantil.

Espera-se que a utilização da tecnologia educacional, composta por diferentes estratégias educativas, possa favorecer a mudança de comportamento voltado para a lavagem das mãos entre as crianças que participaram da ação educativa, no intuito de promover a prevenção e redução de infecções intestinais e respiratórias.

Observa-se que a enfermagem é uma das profissões que mais trabalham com educação em saúde e, por isso, essa experiência foi rica e fundamental para que, como enfermeiros, possamos planejar as ações de educação em saúde direcionadas às necessidades do públicoalvo, utilizando recursos didáticos que podem ser adaptados, utilizando assim a criatividade.

A limitação desta pesquisa está relacionada ao fato de utilizar recursos visuais pré-existentes e adaptados à proposta educativa, além disso, a experiência dos discentes foi em um ambiente privado, durante um curto período dentro do estágio supervisionado em atenção básica à saúde.

\section{REFERÊNCIAS}

1 -Soares AL, Neves EAO, SOUSA IFAC. A importância da educação sanitária no controle e prevenção ao ascaris lumbricoides na infância. Ciênc Biol Saúde Unit 2018 [citado em 11 abr 2021]; 3(3):23-32. Acesso em: https://periodicos.set.edu.br/facipesaude/article Lview/5980

2 - Bieri FA, Gray DJ, Williams GM, Raso G, Li YS, Yuan $L$, et al. Health-education package to prevent worm infections in Chinese schoolchildren. N Engl J Med. 2013;368(17):160312. DOI: $10.1056 /$ NEJMoa1204885

3 - Almeida NRB, Carvalho AS, Moreno CA. Análise parasitológica de contaminantes de origem fecal encontrados em uma Creche do interior da Bahia. Id on Line Rev Mult Psic. 2017; 11(38):642-51.

DOI:

10.14295/idonline.v11i38.930

4 - Faria KF, Mota KCP, Silva CO, Oliveira MM, Araújo IAC, Mendes GG, et al. Ensino em parasitologia: Ação extensionista com crianças em idade escolar. Revista Conexão UEPG 2019;15(3): 294-300. DOI: 10.5212/Rev.Conexao.v.15.i3.0009

5 - Chittleborough CR, Nicholson AL, Young E, Bel $\mathrm{S}$, Campbell R. Implementation of an educational intervention to improve hand washing in primary schools: Process evaluation within a randomised controlled trial. BMC Public Health 2013;13:757. DOI: $\underline{10.1186 / 1471-2458-13-757}$ 
6 - Nicholson JA, Naeeni M, Hoptroff $M$, Matheson JR, Roberts AJ, Taylor D, et al. An investigation of the effects of a hand washing intervention on health outcomes and school absence using a randomised trial in Indian urban communities. Trop Med Int Health 2014; 19(3):284-92. DOI: 10.1111/tmi.12254

7 - Sigaud CHS, Santos BR, Costa P, Toriyama ATM. Promoção da higiene bucal de préescolares: Efeitos de uma intervenção educativa lúdica. Rev Bras Enferm. 2017;70(3):519-25. DOI: 10.1590/0034-7167-2016-0237

8 - Ramos LS, Gomes HALF, Aguiar TCG, Soares RMS, Corrêa MX, Morgan LTF, et al. Instruções de higiene na escola e na sociedade como ação de saúde e prevenção de doenças: Uma revisão bibliográfica. Revista Eletrônica Acervo Saúde 2020;12(10):1-7. DOI: 10.25248/reas.e4558.2020

9 - Nietsche EA, Backes VMS, Colomé CLM, Ceratti RN, Ferraz F. Tecnologias educacionais, assistenciais e gerenciais: Uma reflexão a partir da concepção dos docentes de enfermagem. Rev Latino-Am Enfermagem 2005;13(3):344-53. DOI: 10.1590/S0104-11692005000300009

10 - Brasil. Agência Nacional de Vigilância Sanitária. Higienização das mãos em serviços de saúde. Brasília: Anvisa; 2007.

11 - Miranda DN, March C, Koifman L. Educação e saúde na escola e a contrarreforma do ensino médio: Resistir para não retroceder. Trab Educ Saúde 2019; 17(2):1-22. DOI: 10.1590/19817746-sol00207

12 - Santiago L, Rodrigues MTP, Oliveira Junior AD, Moreira TMM. Implantação do Programa Saúde na escola em Fortaleza-CE: Atuação de equipe da Estratégia Saúde da Família. Rev Bras Enferm. 2012;65(6):1026-9. DOI: 10.1590/S0034$\underline{71672012000600020}$

13 - Mbakaya BC, Lee PH, Lee RLT. Hand hygiene intervention strategies to reduce diarrhoea and respiratory infections among schoolchildren in developing countries: A systematic review. Int J Environ Res Public Health 2017;14(4):371. DOI: 10.3390 / ijerph14040371

14 - Tefera E, Belay T, Mekonnen SK, Zeynudin A, Belachew T. Prevalence and intensity of soil transmitted helminths among school children of Mendera Elementary School, Jimma, Southwest
Ethiopia. Pan Afr Com J. 2017;27:88. DOI: 10.11604/pamj.2017.27.88.8817

15 - Ribeiro FDO, Souza MA, Paula AO, Silva AG, Oliveira AC. Estratégia lúdica para a melhoria de práticas de higienização das mãos entre os profissionais de saúde. Rev Enferm UFPE on Line 2017;11(10):3971-9. DOI: 10.5205/1981-8963v11i10a25207p3971-3979-2017

16 - Rodrigues EN, Alves MSJ, Sobral MSC. O brincar e o aprender na educação infantil. Rev Mult Psic. 2019;13(43):187-96. DOI: 10.14295/idonline.v13i43.1520

17 - Nicácio LA, Davim RMB, Oliveira MB, Camboim JCF, Medeiros HRL, Oliveira SX. Intervenção educativa sobre o mosquito Aedes Aegypti em escolares: Possibilidade para a enfermagem no contexto escolar. Rev Enferm UFPE on Line 2017;11(10):3771-7. DOI: 10.5205/reuol.12834-30982-1-SM.1110201710

18 - Silva MFA, Santos PFBB, Wesp LHS, Silva LLI, Bispo WF. A enfermagem nas instituições de educação infantil: Refletindo sobre essa parceria. Rev Enferm UFPE on Line 2017;11(supl 8):3310-6. DOI: $\quad 10.5205 /$ reuol.11135-99435-1ED.1108sup201716

19 - Sales CCF, Meschial WC, Oliveira MLF. Construção de oficinas pedagógicas para prevenção das intoxicações infantis. Arq Cienc Saúde UNIPAR 2018 [citado em 11 abr 2021]; 22(1):17-22. Acesso em: http://www.revistas.unipar.br/index.php/saude/ article/download/6221/3556

20 - Rangel RF, Backes DS, Ilha S, Siqueira $\mathrm{HCH}$, Martins FDP, Zamberlan C. Cuidado integral: Significados para docentes e discentes de enfermagem. Rev Rene 2017;18(1):43-50. DOI: 10.15253/2175-6783.2017000100007

21 - Vieira FS, Portela NLC, Sousa GC, Costa ES, Oliveira DEP, Neiva MJLM. Inter-relação das ações de educação em saúde no contexto da Estratégia Saúde da Família: Percepções do enfermeiro. Rev Pesqui. 2017;9(4):1139-44. DOI: 10.9789/2175-5361.2017.v9i4. 1139-1144 
Editores Responsáveis:

Juliana Dias Reis Pessalacia

Kellen Rosa Coelho Sbampato

Nota: Este trabalho faz parte de um Relato de Experiência de

Graduação referente ao Estágio Supervisionado I, sendo um dos itens utilizados para avaliação do discente ao final do semestre.

Recebido em: 13/09/2019

Aprovado em: $13 / 04 / 2021$ 